\title{
A Nonlinear Transceiver Architecture for Overloaded Multiuser MIMO Interference Channels
}

\author{
Youngseok Oh, Heejung Yu, Yong H. Lee, and Youngchul Sung
}

\begin{abstract}
A new transceiver architecture for overloaded MIMO interference channels is proposed to fix the rate saturation problem of purely linear beamforming in this case. The proposed scheme is based on a mixture of linear beamforming and multiuser detection. It is shown that non-trivial degrees of freedom can be achieved by the proposed mixed scheme properly dividing the interference signals for linear processing and multiuser detection, and the achievable degrees of freedom of the proposed scheme are obtained. Numerical results show that the proposed scheme outperforms linear beamforming in overloaded MIMO interference channels.
\end{abstract}

Index Terms-Multi-user MIMO, MIMO interference channels, multi-user decoding, successive interference cancellation, overloaded systems.

\section{INTRODUCTION}

$\mathbf{M}$ ULTI-USER multiple-input multiple-output (MIMO) interference channels have extensively been studied recently because many concurrent wireless systems can be modeled in this form. For $K$-user time-varying interference channels, interference alignment (IA) has been introduced as a powerful tool for achieving maximum degrees of freedom (DoF) [1]. Although IA can achieve approximately one half of the capacity that can be achieved in the absence of interference at high signal-to-noise ratio (SNR), it has loss in the sum rate at low and intermediate SNR, and thus, several algorithms based on linear beamforming have been proposed for MIMO interference channels to maximize the sum rate [2]-[5]. These linear-beamforming-based algorithms yield higher sum rates, especially at low and intermediate SNR, than IA, but their sum rates also saturate in time-invariant overloaded ${ }^{1}$ MIMO systems as SNR increases. (That is, the DoF of such a system with linear beamforming is zero.) This is because the achievable DoF of these algorithms based on linear beamforming is the same as that of IA [6].

The rate saturation problem of an overloaded MIMO interference system can be resolved simply by using a scheduler. For an overloaded example of four users and two antennas,

Paper approved by N. Al-Dhahir, the Editor for Space-Time, OFDM and Equalization of the IEEE Communications Society. Manuscript received June 17, 2011; revised September 7, and November 23, 2011.

Y. Oh, Y. H. Lee, and Y. Sung (corresponding author) are with the Department of Electrical Engineering, KAIST, South Korea, 305-701 (e-mail: \{ysoh@stein,yohlee@ee, ysung@ee\}.kaist.ac.kr).

H. Yu is with the ETRI, Daejeon, South Korea, 305-700 (e-mail: heejung@etri.re.kr).

This research was supported by the KCC (Korea Communications Commission), Korea, under the R\&D program supervised by the KCA (Korea Communications Agency) (KCA-2011-08913-04001).

Digital Object Identifier 10.1109/TCOMM.2012.022912.110130

${ }^{1}$ We define an overloaded MIMO interference system as a system in which IA is not feasible even for one data stream per user. In this paper, we consider the practical signal-space interference alignment only.

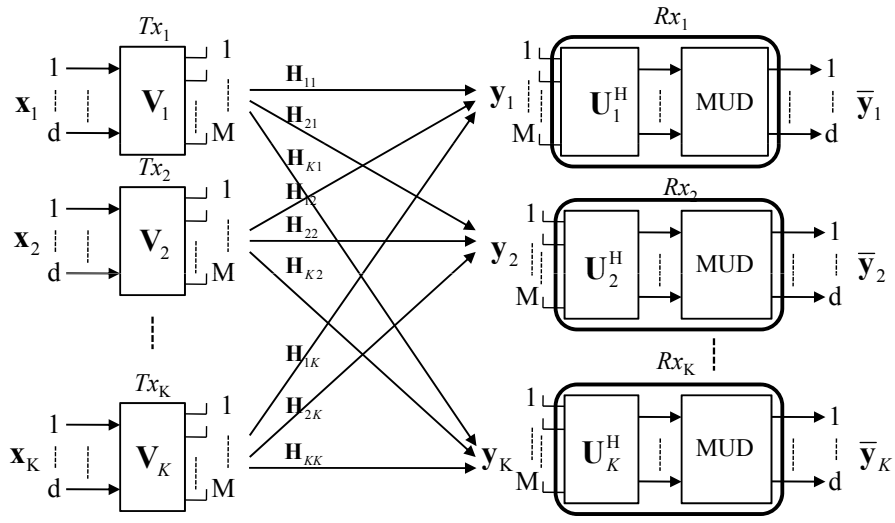

Fig. 1. A $K$-user $M \times M$ MIMO interference channel model.

DoF three instead of zero can be achieved by a simple roundrobin scheduler that allows only three transmitters to transmit at a time. However, using a scheduler requires coordination among transmitters. In this paper, we propose a transceiver architecture based on nonlinear processing to enhance the sum-rate performance for time-invariant overloaded MIMO interference channels based on receiver processing only when such scheduling among the transmitters is not available. Motivated by the transceiver scheme in two-user interference channels with weak interference [7], at each receiver we treat some interference signals as signals to be decoded simultaneously with the desired signal by multi-user decoding (MUD), while treating other interference signals as interference to be suppressed by a linear receive filter. We propose an exhaustive design method to maximize the sum rate under the proposed system architecture, and propose a simplified algorithm based on a person-by-person optimization approach to reduce computational complexity. In the case of using successive interference cancellation (SIC) as MUD, we derive a strategy and the corresponding DoF for the proposed nonlinear processing scheme. Even for a overloaded MIMO interference channel, using the proposed scheme a nonzero DoF for the overall system can be obtained without any scheduler unlike the systems based on linear beamforming. The performance of the proposed architecture is examined numerically. The results show that the proposed system architecture can achieve a much higher sum rate than the system based only on linear beamforming in the overloaded case.

\section{SySTEM MODEL}

We consider a time-invariant $K$-user $M \times M$ MIMO interference channel, as shown in Fig. 1, where all transmitters and receivers are equipped with $M$ antennas per each $(M \geq 2)$. 
Each transmitter paired with a corresponding receiver interferes with all the unpaired receivers. Transmitter $k$ sends a complex-valued data vector $\mathbf{x}_{k} \in \mathbb{C}^{d \times 1}$ with power (or norm square) $P$ after preprocessing by $\mathbf{V}_{k} \in \mathbb{C}^{M \times d}$, where each stream of $\mathbf{x}_{k}$ is encoded from a Gaussian codebook, i.e., $\mathbf{x}_{k} \sim \mathcal{C N}\left(0, \frac{P}{d} \mathbf{I}_{d}\right) ; d$ represents the number of streams to be sent from each transmitter; $k \in \mathcal{K}:=\{1,2, \ldots, K\} ; \mathbf{I}_{d}$ denotes a $d \times d$ identity matrix and $\mathbf{V}_{k}$ is the precoder matrix. The time-invariant flat-fading MIMO channel from transmitter $j$ to receiver $k$ is denoted as $\mathbf{H}_{k j} \in \mathbb{C}^{M \times M}$ for $k, j \in \mathcal{K}$, where the $(m, n)$ th entry of $\mathbf{H}_{k j}$ represents the complex channel gain from the $n$th antenna of transmitter $j$ to the $m$ th antenna of receiver $k$ and is assumed to be a realization of an independent and identically distributed (i.i.d) complex Gaussian random variable with zero mean and unit variance. We assume that the system is overloaded, i.e., $K \geq 2 M$ for $M \times M$ MIMO systems [6], and each terminal has all the channel information. At each receiver, $\left(K-1-N_{M U D}\right)$ signals among all $K-1$ interference signals are suppressed by a linear receive filtering matrix $\mathbf{U}_{k} \in \mathbb{C}^{M \times d}$ and the $N_{M U D}$ remaining interference signals pass through the linear receiver filter and are then jointly decoded by MUD together with the desired signal. Then, the received signal $\mathbf{y}_{k} \in \mathbb{C}^{M \times 1}$ at receiver $k$ is given by

$$
\mathbf{y}_{k}=\mathbf{H}_{k k} \mathbf{V}_{k} \mathbf{x}_{k}+\sum_{j \in \mathcal{S}_{k} \backslash\{k\}} \mathbf{H}_{k j} \mathbf{V}_{j} \mathbf{x}_{j}+\sum_{j \notin \mathcal{S}_{k}} \mathbf{H}_{k j} \mathbf{V}_{j} \mathbf{x}_{j}+\mathbf{z}_{k},
$$

where $\mathcal{S}_{k}$ is the set of indices of the transmitters whose signals are decoded by MUD at receiver $k$ and $\mathbf{z}_{k} \in \mathbb{C}^{M \times 1}$ is additive white Gaussian noise ${ }^{2}$ (AWGN) with $\mathbf{z}_{k} \sim \mathcal{C N}\left(0, \mathbf{I}_{M}\right)$. Here, $k \in \mathcal{S}_{k}$ and $\left|\mathcal{S}_{k}\right|=N_{M U D}+1$. The third and fourth terms on the right-hand-side (RHS) of (1) are to be suppressed by the linear receive filter $\mathbf{U}_{k}$. The $N_{M U D}+1$ transmitters in $\mathcal{S}_{k}$ and receiver $k$ form a multiple access channel (MAC), which will be referred to as the $k$ th $M A C$ hereafter. Given $\mathcal{S}_{k}, P$, and $\left\{\mathbf{H}_{k j}\right\}$, the transmission rates under the proposed scheme should reside within the capacity region of the $k$ th MAC for each $k$ for successful transmission. That is, let $\left\{R_{1}\left(P,\left\{\mathbf{H}_{k j}\right\}\right), \cdots, R_{K}\left(P,\left\{\mathbf{H}_{k j}\right\}\right)\right\}$ denote the transmission rates of all $K$ transmitters for given $\left(P,\left\{\mathbf{H}_{k j}\right\}\right)$, and let $\mathcal{R}_{k}\left(P,\left\{\mathbf{H}_{k j}\right\}\right)$ denote the capacity region of the $k$ th MAC. Then, for successful transmission we have

$$
\left(R_{1}\left(P,\left\{\mathbf{H}_{k j}\right\}\right), \ldots, R_{K}\left(P,\left\{\mathbf{H}_{k j}\right\}\right)\right) \in \bigcap_{k=1}^{K} \mathcal{R}_{k}\left(P,\left\{\mathbf{H}_{k j}\right\}\right) .
$$

\section{DESIGN AND ANALYSIS}

In this section we optimize the parameters $d, N_{M U D},\left\{\mathcal{S}_{k}\right\}$, $\left\{\mathbf{V}_{j}\right\},\left\{\mathbf{U}_{k}\right\}$, and $\left\{R_{j}\right\}$ to maximize the sum rate of the proposed nonlinear scheme. We first consider an exhaustive search to optimize the design parameters of the proposed

\footnotetext{
${ }^{2}$ To simplify analysis, we neglect different path losses and shadowing terms for the channel links, and assume that all of the channels are i.i.d and each receiver has the same noise power. Under this assumption, all receivers in the network have the same average SNR from all transmitters. This is a reasonable and practical assumption under a cellular infrastructure in which the users experiencing inter-cell interference are typically in the cell edge and thus have similar average SNR.
}

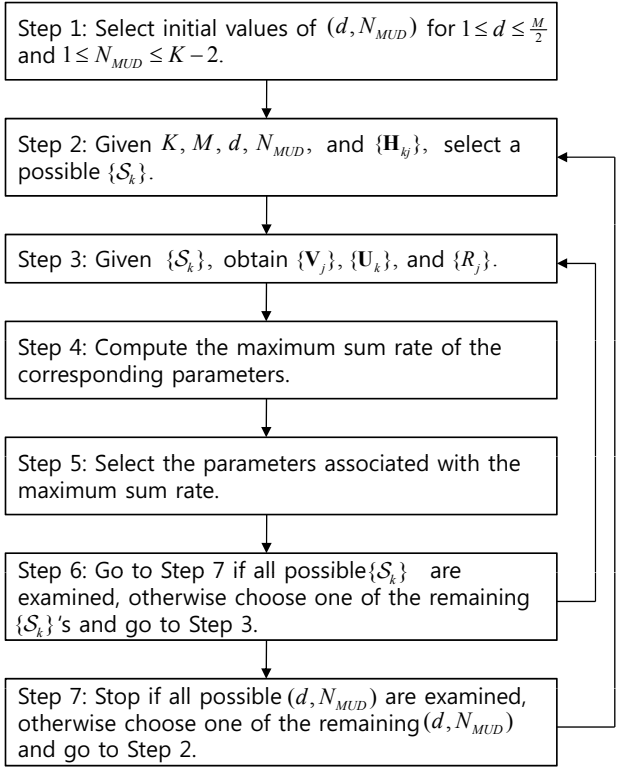

Fig. 2. Flow-chart for designing the proposed nonlinear system. The inner and outer loops need $\left(\begin{array}{c}K-1 \\ N_{M U D}\end{array}\right)^{K}$ and $\frac{M}{2}(K-2)$ iterations, respectively.

scheme. The procedure to obtain optimal $d, N_{M U D}$, and $\left\{\mathcal{S}_{k}\right\}$ for the proposed scheme is described in Fig. 2. For each feasible combination of $\left(d, N_{M U D},\left\{\mathcal{S}_{k}\right\}\right)$, the parameters $\left\{\mathbf{V}_{k}, \mathbf{U}_{k}, k=1, \cdots, K\right\}$, and rate-tuple $\left\{R_{k}, k=1, \cdots, K\right\}$ are obtained, and the corresponding sum rate is evaluated and stored with the associated parameters. The parameters associated with the maximum sum rate are selected. From here on we explain each step in detail and analyze the performance of the proposed scheme.

\section{A. Design of precoders and receive filters}

Given $\left(d, N_{M U D}\right)$ and $\left\{\mathcal{S}_{k}\right\}$, the linear beamforming matrices $\left\{\mathbf{V}_{j}\right\}$ and $\left\{\mathbf{U}_{k}\right\}$ are obtained by modifying one of the existing linear beamformer design algorithms [5]. We here consider the Max-SINR algorithm [2] which is shown to be optimal at high SNR [5]. In the modified algorithm, the interference-and-noise covariance matrix for the whitened matched (or minimum mean square error (MMSE)) filtering at receiver $k$ is given by

$$
\mathbf{Q}_{k}=\frac{P}{d} \sum_{j \notin \mathcal{S}_{k}} \mathbf{H}_{k j} \mathbf{V}_{j} \mathbf{V}_{j}^{H} \mathbf{H}_{k j}^{H}+\mathbf{I}_{M},
$$

and the receiver filter $\mathbf{U}_{k}=\alpha \mathbf{Q}_{k}^{-1} \mathbf{H}_{k k} \mathbf{V}_{k}$ with a normalization factor $\alpha$ at receiver $k$ is designed to suppress the interference from the transmitters not contained in $\mathcal{S}_{k}$ and noise. The precoders $\left\{\mathbf{V}_{j}\right\}$ are designed based on channel reciprocity. That is, $\mathbf{V}_{j}$ is determined as the whitened matched filter with the interference-and-noise covariance matrix $\overleftarrow{\mathbf{Q}}_{j}=$ $\frac{P}{d} \sum_{k \in \mathcal{K} \backslash \mathcal{D}_{j}} \overleftarrow{\mathbf{H}}_{j k} \mathbf{U}_{k} \mathbf{U}_{k}^{H} \overleftarrow{\mathbf{H}}_{j k}^{H}+\mathbf{I}_{M}$, where $\overleftarrow{\mathbf{H}}_{j k}=\mathbf{H}_{j k}^{H}$ due to channel reciprocity as in [2] and $\mathcal{D}_{j}=\left\{k \mid j \in \mathcal{S}_{k}\right\}$, i.e., the index set of receivers that decode the signal of transmitter $j$. Thus, in the modified algorithm other users' signals that are jointly decoded by MUD are not included in the interference-and-noise covariance, and the resulting $\left\{\mathbf{V}_{j}\right\}$ 
and $\left\{\mathbf{U}_{k}\right\}$ suppress the interference signals not contained in $\mathcal{S}_{k}$ only. When $\mathcal{S}_{k}=\{k\}$ for all $k$, the modified algorithm reduces to the original Max-SINR algorithm. At high SNR, the modified algorithm obtains beamforming matrices satisfying ${ }^{3}$

$$
\mathbf{U}_{k}^{H} \mathbf{H}_{k j} \mathbf{V}_{j}=\mathbf{0}_{d} \quad \text { for } \quad\left\{(j, k) \mid j \neq k, j \notin \mathcal{S}_{k}, j, k \in \mathcal{K}\right\}
$$

(not for $\{(j, k) \mid j \neq k, j, k \in \mathcal{K}\}$ as in the original IA scheme), where $\mathbf{0}_{d}$ denotes a $d \times d$ all-zero matrix. Note that a solution to (4) for $\{(j, k) \mid j \neq k, j, k \in \mathcal{K}\}$ does not exist in the overloaded case. However, a solution to (4) for $\left\{(j, k) \mid j \neq k, j \notin \mathcal{S}_{k}, j, k \in \mathcal{K}\right\}$ exists even in the overloaded case if $\mathcal{S}_{k}$ are designed properly. This result is summarized in the following proposition.

Proposition 1: In the proposed nonlinear scheme, $\left\{\mathbf{V}_{j}\right\}$ and $\left\{\mathbf{U}_{k}\right\}$ satisfying (4) for $\left\{(j, k) \mid j \neq k, j \notin \mathcal{S}_{k}, j, k \in \mathcal{K}\right\}$ exist if

$d \leq \min \left\{\frac{M}{2}, \bar{D}\right\}$, where $\bar{D}=\frac{\left(2 K-N_{C O M}\right) M}{K\left(K+1-N_{M U D}\right)-N_{C O M}}$

and $N_{C O M}=\left|\left\{j \mid j \in \mathcal{K}, j \in \bigcap_{k=1}^{K} \mathcal{S}_{k}\right\}\right|$, i.e., the number of transmitters commonly contained in all $\mathcal{S}_{k}, k \in \mathcal{K}$.

Proof: The proof is similar to that for the general IA condition in [6]. The condition $d \leq M / 2$ is trivial. Thus, we focus on the second condition. $\left\{\mathbf{V}_{j}\right\}$ and $\left\{\mathbf{U}_{k}\right\}$ satisfying (4) exist if and only if $N_{v} \geq N_{e}$, where $N_{v}$ and $N_{e}$ are the numbers of variables and equations in (4), respectively. In (4), each $\mathbf{U}_{k}^{H} \mathbf{H}_{k j} \mathbf{V}_{j}$ has $d^{2}$ equations, and for a given $k$ the number of $j$ 's not in $\mathcal{S}_{k}$ is $\left(K-1-N_{M U D}\right)$. Thus, $N_{e}=K\left(K-1-N_{M U D}\right) d^{2}$. On the other hand, $\left\{\mathbf{V}_{j}\right\}$ and $\left\{\mathbf{U}_{k}\right\}$ satisfying (4) consist of $d$ linearly independent column vectors. Such independent vectors can be obtained by setting the first $d$ rows of $\mathbf{V}_{j}$ and $\mathbf{U}_{k}$ as the $d \times d$ identity matrix. Hence, the number of independent variables of $\mathbf{V}_{j}$ ( or $\mathbf{U}_{k}$ ) is $(M-d) d$. Since the numbers of $\mathbf{V}_{j}$ and $\mathbf{U}_{k}$ to be determined are $K-N_{C O M}$ and $K$, respectively (if $j \in \bigcap_{k=1}^{K} \mathcal{S}_{k}, \mathbf{V}_{j}$ does not appear in (4)), we have $N_{v}=\left(2 K-N_{C O M}\right)(M-d) d$. Now applying $N_{v} \geq N_{e}$ yields $d \leq \bar{D}$.

Corollary 1: We have

$$
\lim _{K \rightarrow \infty} \bar{D}=\left\{\begin{array}{rr}
0 & \text { if } N_{M U D} \text { is a constant, } \\
\frac{M}{C_{I}} \quad, & \text { if } N_{M U D}=K-C_{I} \text { and } \\
N_{C O M}=K-C_{C}, \\
\frac{2 M}{C_{I}+1}, & \text { if } N_{M U D}=K-C_{I} \text { and } \\
N_{C O M} \text { is a constant }
\end{array}\right.
$$

for some positive integers $C_{I}$ and $C_{C}$.

Proof: For each case, substitute the corresponding $\left(N_{M U D}, N_{C O M}\right)$ into $\bar{D}$ in (5), take limit $\lim _{K \rightarrow \infty} \bar{D}$, and apply the L'Hopital's Rule. Then, we obtain the result.

Corollary 2: Given $K, M$, and $N_{M U D}, \bar{D}$ monotonically decreases as $N_{C O M}$ increases from zero to $N_{M U D}$.

\footnotetext{
${ }^{3}$ It was shown that the solution (or fixed point) to the Max-SINR algorithm satisfies the interference alignment property at high SNR [5]. (4) means that we require the linear processing part to suppress the interference not contained in $\mathcal{S}_{k}$ fully.
}

Proof: $\bar{D}$ in (5) can be written as $\bar{D}\left(N_{C O M}\right)=$ $\frac{A-N_{C O M} M}{B-N_{C O M}} \quad(\geq 0)$, where $A=2 K M$ and $B=K(K+$ $\left.1-N_{M U D}\right)$. Let $\alpha=N_{C O M}$ and let $\beta=N_{C O M}+1$. $(\alpha A+\beta B M)-(\alpha B M+\beta A)=(\beta-\alpha)(B M-A) \geq 0$ since $\alpha<\beta$ and $B M \geq A$, $\left(N_{M U D} \leq K-1\right)$. Then, $\frac{\bar{D}\left(N_{C O M}\right)}{\overline{D\left(N_{C O M}+1\right)}}=\frac{\bar{D}(\alpha)}{\bar{D}(\beta)}=\frac{\bar{A} B+\alpha \beta M-(\alpha B M+\beta A)}{A B+\alpha \beta M-(\alpha A+\beta B M)} \geq 1$ for all $N_{C O M}$, and thus the claim follows.

Corollaries 1 and 2 provide a strategy to design $\left\{\mathcal{S}_{k}\right\}$ optimally for the proposed scheme. Since $\frac{2 M}{C_{I}+1} \geq \frac{M}{C_{I}}$ in (6) for $C_{I} \geq$ 1 , to maximize $\bar{D}$, we should increase $N_{M U D}$ linearly with $K$ while keeping $N_{C O M}$ constant by Corollary 1 . Also, by Corollary 2, we should have $N_{C O M}=0$ to maximize $\bar{D}$. Note that, in the purely linear beamforming case, we have $N_{M U D}=N_{C O M}=0$, and thus, $\bar{D}=2 M /(K+1)$ is strictly less than one in the overloaded case $K \geq 2 M$, which implies $d=0$. On the contrary, the proposed nonlinear scheme yields a non-zero DoF in the overloaded case by designing $N_{M U D}$ and $N_{C O M}$ properly.

\section{B. Data Rates and Achievable DoF}

For the given $\left(d, N_{M U D},\left\{\mathcal{S}_{k}\right\}\right)$ and $\left\{\mathbf{V}_{k}, \mathbf{U}_{k}\right\}$ obtained in the previous subsection, the input to MUD at receiver $k$ is given by $\overline{\mathbf{y}}_{k}=\mathbf{U}_{k}^{H} \mathbf{y}_{k}$ and the capacity region $\mathcal{R}_{k}\left(P,\left\{\mathbf{H}_{k j}\right\}\right)$ of the $k$ th MAC is determined by a set of inequalities[8]

$$
\begin{aligned}
\sum_{j \in \mathcal{S}_{k, i}} R_{j}\left(P,\left\{\mathbf{H}_{k j}\right\}\right) & \leq I\left(\mathbf{x}\left(\mathcal{S}_{k, i}\right) ; \overline{\mathbf{y}}_{k} \mid \mathbf{x}\left(\mathcal{S}_{k, i}^{c}\right)\right) \\
& =\log \operatorname{det}\left(\mathbf{I}+\mathbf{Q}_{k}^{-1} \frac{P}{d} \sum_{j \in \mathcal{S}_{k, i}} \mathbf{H}_{k j} \mathbf{T}_{j} \mathbf{H}_{k j}^{H}\right)
\end{aligned}
$$

for each $k \in \mathcal{K}$, where $\mathcal{S}_{k, i}, i=1, \ldots, 2^{N_{M U D}+1}-1$, are all subsets of $\mathcal{S}_{k}$ except the empty set, $\mathbf{x}\left(\mathcal{S}_{k, i}\right):=\left\{\mathbf{x}_{j} ; j \in \mathcal{S}_{k, i}\right\}$, $\mathbf{x}\left(\mathcal{S}_{k, i}^{c}\right):=\left\{\mathbf{x}_{j} ; j \in \mathcal{S}_{k, i}^{c}\right\}, \mathbf{Q}_{k}$ is the interference-and-noise covariance matrix defined in (3), and $\mathbf{T}_{j}=\mathbf{V}_{j} \mathbf{V}_{j}^{H}, j \in \mathcal{S}_{k, i}$. For successful transmission, the inequalities in (7) should be satisfied for all $k$, and the rates maximizing the sum rate can be obtained by solving the following linear programming (LP) problem:

$$
\begin{array}{ll}
\operatorname{maximize} & \sum_{j=1}^{K} R_{j}\left(P,\left\{\mathbf{H}_{k j}\right\}\right) \\
\text { subject to } & \text { the inequalities in (7) for all } k \in \mathcal{K} .
\end{array}
$$

To simplify the analysis, from here on we consider successive interference cancellation (SIC) whose performance serves as a lower bound for general MUD. Since the performance of SIC depends on the decoding order, we define the decoding order $\pi_{k}$ for $\mathcal{S}_{k}$ at receiver $k$. We also define $\mathcal{M}_{j}^{\left(\pi_{k}\right)}$ as the subset of $\mathcal{S}_{k}$ containing the indices of all transmitters whose data are decoded before transmitter $j$ under the decoding order $\pi_{k}$. Then, the rate for transmitter $j\left(\in \mathcal{S}_{k}\right)$ to receiver $k$ under SIC with the decoding order $\pi_{k}$ is bounded by

$$
\begin{aligned}
R_{j}^{S I C\left(\pi_{k}\right)}\left(P,\left\{\mathbf{H}_{k j}\right\}\right) & \leq I\left(\mathbf{x}_{j} ; \overline{\mathbf{y}}_{k} \mid \mathbf{x}\left(\mathcal{M}_{j}^{\left(\pi_{k}\right)}\right)\right) \\
& =: \bar{I}_{k j}^{\left(\pi_{k}\right)}\left(P,\left\{\mathbf{H}_{k j}\right\}\right)
\end{aligned}
$$

for sufficiently large transmission block length. The rate in (10) represents a corner point of the capacity region of the $k$ th MAC associated with $\mathcal{S}_{k}$ and $\pi_{k}$. The maximum rate of 
transmitter $j$ for reliable transmission under the decoding order $\left\{\pi_{k}\right\}$ is given by

$$
\bar{R}_{j}^{\left(\pi_{1}, \cdots, \pi_{K}\right)}\left(P,\left\{\mathbf{H}_{k j}\right\}\right)=\min _{k \in \mathcal{D}_{j}}\left\{\bar{I}_{k j}^{\left(\pi_{k}\right)}\left(P,\left\{\mathbf{H}_{k j}\right\}\right)\right\} .
$$

Then, the optimal SIC decoding order is given by

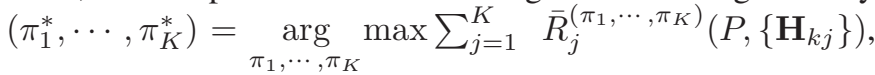
and the optimal rate under SIC for given $\left(d, N_{M U D}\right)$ and $\left\{\mathcal{S}_{k}\right\}$ is given by

$$
R_{j}^{*}\left(P,\left\{\mathbf{H}_{k j}\right\}\right)=\min _{k \in \mathcal{D}_{j}}\left\{\bar{I}_{k j}^{\left(\pi_{k}^{*}\right)}\left(P,\left\{\mathbf{H}_{k j}\right\}\right)\right\} .
$$

The total DoF of the proposed scheme with SIC is now given by

$$
\begin{aligned}
\Gamma_{\text {SIC }} & =\lim _{P \rightarrow \infty} \frac{\sum_{j=1}^{K} R_{j}^{*}\left(P,\left\{\mathbf{H}_{k j}\right\}\right)}{\log P} \\
& =\lim _{P \rightarrow \infty} \frac{\sum_{j=1}^{K} \min _{k \in \mathcal{D}_{j}}\left\{\bar{I}_{k j}^{\left(\pi_{k}^{*}\right)}\left(P,\left\{\mathbf{H}_{k j}\right\}\right)\right\}}{\log P} \\
& =\sum_{j=1}^{K} \min _{k \in \mathcal{D}_{j}} \Gamma_{k j},
\end{aligned}
$$

where $\Gamma_{k j}:=\lim _{P \rightarrow \infty} \bar{I}_{k j}^{\left(\pi_{k}^{*}\right)}\left(P,\left\{\mathbf{H}_{k j}\right\}\right) / \log (P)$, because the limit, summation, and min operation are order-independent when the number of terms is finite. $\Gamma_{k j}$ can easily be obtained by noting that $\bar{I}_{k j}^{\left(\pi_{k}^{*}\right)}\left(P,\left\{\mathbf{H}_{k j}\right\}\right)$ is in the following form:

$$
\bar{I}_{k j}^{\left(\pi_{k}^{*}\right)}\left(P,\left\{\mathbf{H}_{k j}\right\}\right)= \begin{cases}\sum_{i=1}^{d} \log \left(1+P_{k j}^{S}\right), \text { if } \mathcal{M}_{j}^{\left(\pi_{k}^{*}\right)}=\mathcal{S}_{k} \backslash\{j\}, \\ \sum_{i=1}^{d} \log \left(1+\frac{P_{k j}^{S}}{1+P_{k j}^{I}}\right), & \text { otherwise, }\end{cases}
$$

where $P_{k j}^{S}$ is the power of transmitter $j$ 's signal and $P_{k j}^{I}$ is that of the interference not cancelled yet at the stage of decoding transmitter $j$. Thus, only the transmitter that is decoded at the last decoding stage at each receiver has a non-zero degree of freedom, i.e.,

$$
\Gamma_{k j}= \begin{cases}d, & \text { if } \mathcal{M}_{j}^{\left(\pi_{k}^{*}\right)}=\mathcal{S}_{k} \backslash\{j\}, \\ 0, & \text { otherwise. }\end{cases}
$$

If the signal of transmitter $j$ is not decoded at the last stage at all receivers in $\mathcal{D}_{j}$, then $\Gamma_{k j}=0$ for some $k \in \mathcal{D}_{j}$, and thus $\min \left\{\Gamma_{k j}, k \in \mathcal{D}_{j}\right\}$ is zero in the proposed scheme with SIC. Let $N_{\Gamma 0}$ be the number of such transmitters. Then, by (13) and (15), $\Gamma_{\text {SIC }}$ is given by

$$
\Gamma_{\text {SIC }}=\left(K-N_{\Gamma 0}\right) d,
$$

because $N_{\Gamma 0}$ users have zero DoF and all the other $K-N_{\Gamma 0}$ users have $d$ DoFs. Since $\left|\mathcal{S}_{k}\right|=N_{M U D}+1$, we have $\left|\left\{\Gamma_{k j} \mid 1 \leq j \leq K, k \in \mathcal{D}_{j}\right\}\right|=K\left(N_{M U D}+1\right)$. From (15), only $K$ elements of $\left\{\Gamma_{k j} \mid 1 \leq j \leq K, k \in \mathcal{D}_{j}\right\}$ are equal to $d$. Hence, the set $\left\{\Gamma_{k j} \mid \Gamma_{k j}=0,1 \leq j \leq K, k \in \mathcal{D}_{j}\right\}$ has $K N_{M U D}$ elements. It is seen from (16) that we should minimize $N_{\Gamma 0}$ while maximizing $d$ to maximize $\Gamma_{\text {SIC }}$. In the following, we obtain an upper bound of $\Gamma_{\text {SIC }}$ using the upper bound (5) of $d$ and a lower bound of $N_{\Gamma 0}$ given in Lemma 1.
Lemma 1: $N_{Г 0}$ satisfies the following inequality:

$$
N_{\Gamma 0} \geq \frac{K N_{M U D}-N_{C O M}}{K-1} .
$$

Proof: As mentioned earlier, there are a total of $K\left(N_{M U D}+1\right)$ MUD links in the system, and among them, only $K$ links have DoF $d$. Thus, $K N_{M U D}$ links of DoF zero should be covered by $N_{\Gamma 0}$ transmitters with DoF zero. To minimize $N_{\Gamma 0}$, each transmitter with DoF zero should cover the maximum number of links of DoF zero. Thus, all $N_{C O M}$ transmitters decoded at all receivers should be included in the null-DoF-user set, and the remaining $N_{\Gamma 0}-N_{C O M}$ users should also cover links with DoF zero maximally. In this way, the best strategy yields

$$
K N_{C O M}+(K-1)\left(N_{\Gamma 0}-N_{C O M}\right) \geq K N_{M U D},
$$

which results in (17).

Based on (16), (17), and (5), the total DoF $\Gamma_{\text {SIC }}$ is maximized when

$$
N_{\Gamma 0}=\left\lceil\frac{K N_{M U D}-N_{C O M}}{K-1}\right\rceil
$$

and

$$
d=\min \left\{\left\lfloor\frac{M}{2}\right\rfloor,\left\lfloor\frac{\left(2 K-N_{C O M}\right) M}{K\left(K+1-N_{M U D}\right)-N_{C O M}}\right\rfloor\right\},
$$

where $\lceil\cdot\rceil$ and $\lfloor\cdot\rfloor$ are ceil and floor operations, respectively. When we set $N_{M U D}=K-C_{I}$ and $N_{C O M}=0$, as suggested by Corollaries 1 and 2, we have

$$
\begin{aligned}
N_{\Gamma 0} & =\left\lceil K-\left(C_{I}-1\right)+\frac{1-C_{I}}{K-1}\right\rceil \\
& =\left\lceil N_{M U D}+1+\frac{1-C_{I}}{K-1}\right\rceil \\
& =N_{M U D}+1,
\end{aligned}
$$

where the third equality holds because $C_{I} \leq K-1$. The achievable DoF by the proposed mixed scheme in the overloaded case is given by the following proposition.

Proposition 2: Suppose that $K \geq 2 M$ (overloaded), $N_{M U D}=K-C_{I}$ and $N_{C O M}=0$. (This $\left(N_{M U D}, N_{C O M}\right)$ pair maximizes $d$ in (19) by Corollaries 1 and 2). Then, $\Gamma_{\text {SIC }}$ is maximized if $C_{I}=2 M-1$, and the maximum total DoF is given by

$$
\Gamma_{\text {SIC }}^{*}=2 M-2 .
$$

Proof: From (16), (19), and (20), $\Gamma_{\mathrm{SIC}}=\left(K-N_{\Gamma 0}\right) d=$ $\left(K-N_{M U D}-1\right)\left\lfloor\frac{2 K M}{K\left(K+1-N_{M U D}\right)}\right\rfloor=\left(C_{I}-1\right)\left\lfloor\frac{2 M}{C_{I}+1}\right\rfloor$. Let $\left\lfloor\frac{2 M}{C_{I}+1}\right\rfloor=\iota$, where $\iota$ is a non-negative integer. The upper and lower bounds of $C_{I}$ can then be obtained in terms of $\iota$ :

$$
\frac{2 M-\iota-1}{\iota+1}<C_{I} \leq \frac{2 M-\iota}{\iota} .
$$

Now suppose that $\iota=1$. Then, $M-1<C_{I} \leq 2 M-1$ and $\Gamma_{\text {SIC }}=C_{I}-1$. In this case $\Gamma_{\text {SIC }}$ is maximized when $C_{I}=2 M-1$ and the maximum total DoF is $2 M-2$. When $\iota=2$, we have $\frac{2 M}{3}-1<C_{I} \leq M-1$ and $\Gamma_{\text {SIC }}=2\left(C_{I}-1\right)$; $\Gamma_{\mathrm{SIC}}$ is maximized for $C_{I}=M-1$ and the maximum is $2 M-4$. In this manner, we can show that the maximum of $\Gamma_{\text {SIC }}$ decreases as $\iota$ increases. Therefore, $\Gamma_{\text {SIC }}^{*}$ is obtained for $\iota=1$ and given by (21). 


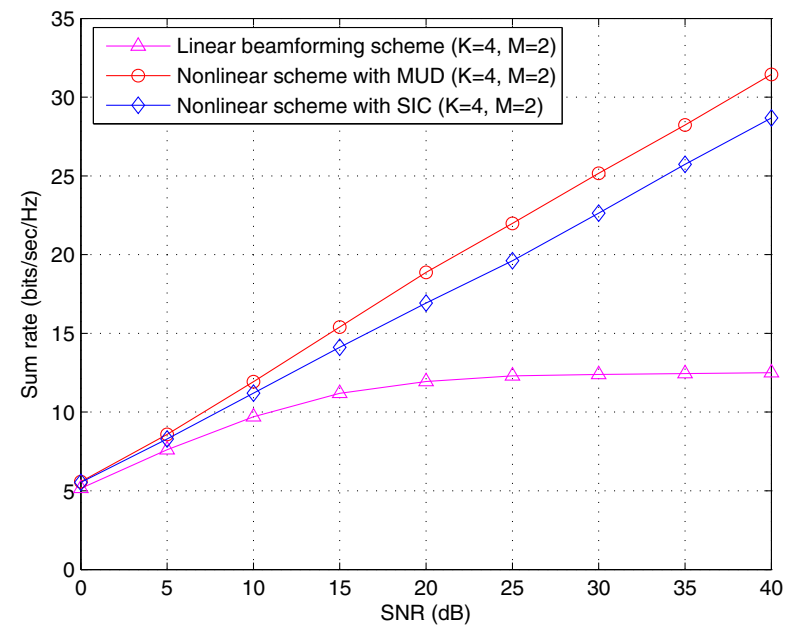

(a)

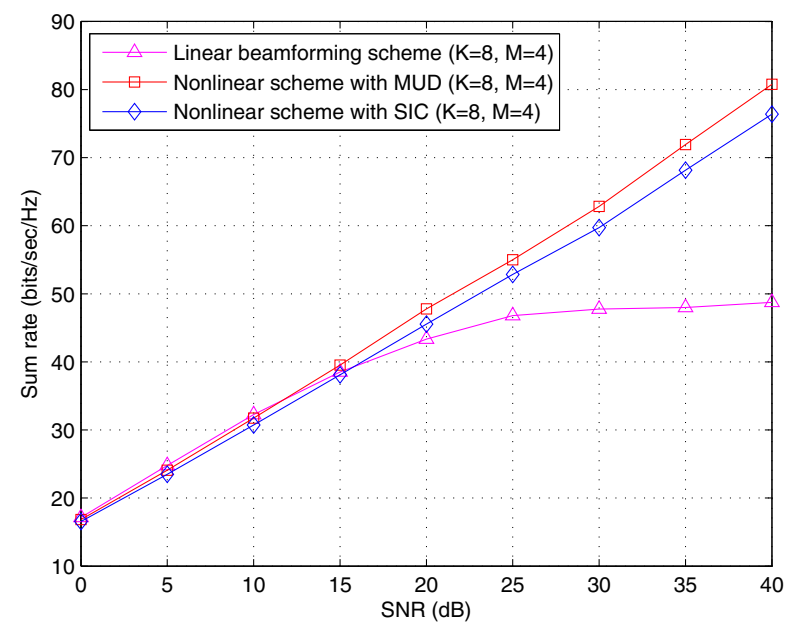

(b)

Fig. 3. Sum rates of the proposed scheme and the linear scheme based on the Max-SINR beamformer design algorithm: (a) $(K, M)=(4,2)$ and (b) $(K, M)=(8,4)$.

For the example of $K=4$ and $M=2$, by Proposition 2, the best user partition for the proposed mixed scheme in the DoF sense is that one of three interfering transmitters is jointly decoded with the desired transmitter $\left(N_{M U D}=1\right)$ and the other two interfering signals are suppressed by the front-end linear beamforming, and this partition yields DoF 2. Note that the best DoF of the proposed scheme is less than three by the round-robin scheduler mentioned in the introduction. This loss is caused by not having transmit coordination.

\section{A Simplified Design Approach to the Proposed Scheme}

For a given $\left(d, N_{M U D}\right)$ the number of all possible $\left\{\mathcal{S}_{k}\right\}$ is $\left(\begin{array}{c}K-1 \\ N_{M U D}\end{array}\right)^{K}$ because there are $K$ users and $N_{M U D}$ signals are selected from $K-1$ interference signals at each receiver. Since the number of all possible pairs of $\left(d, N_{M U D}\right)$ is $\frac{M}{2}(K-2)$ for an even $M$, the exhaustive search for the design of the proposed scheme requires complexity with the order of $\frac{M}{2}(K-2)\left(\begin{array}{c}K-1 \\ N_{M U D}\end{array}\right)^{K}$. Since most of the complexity lies in finding optimal $\left\{\mathcal{S}_{k}\right\}$ for a given $\left(d, N_{M U D}\right)$, the complexity

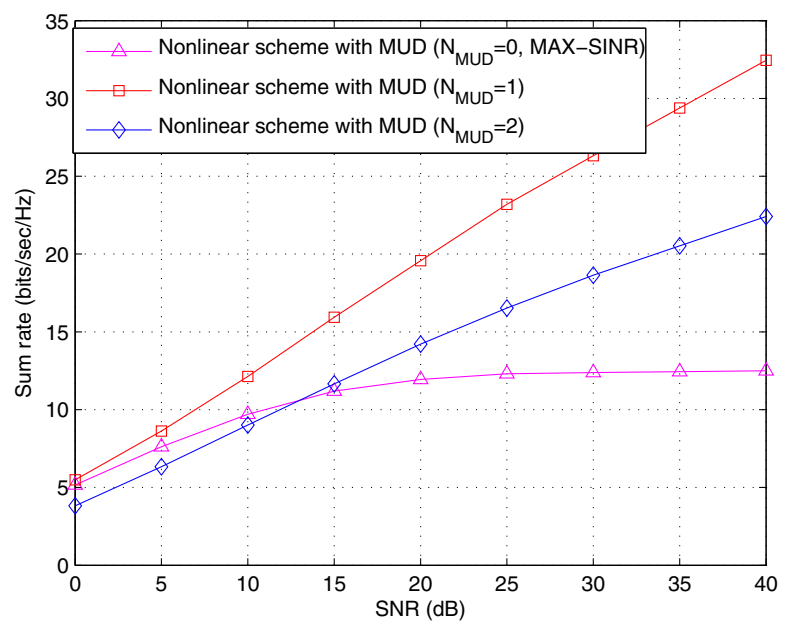

Fig. 4. Sum rates of the proposed scheme with MUD for different values of $N_{M U D}((K, M)=(4,2))$.

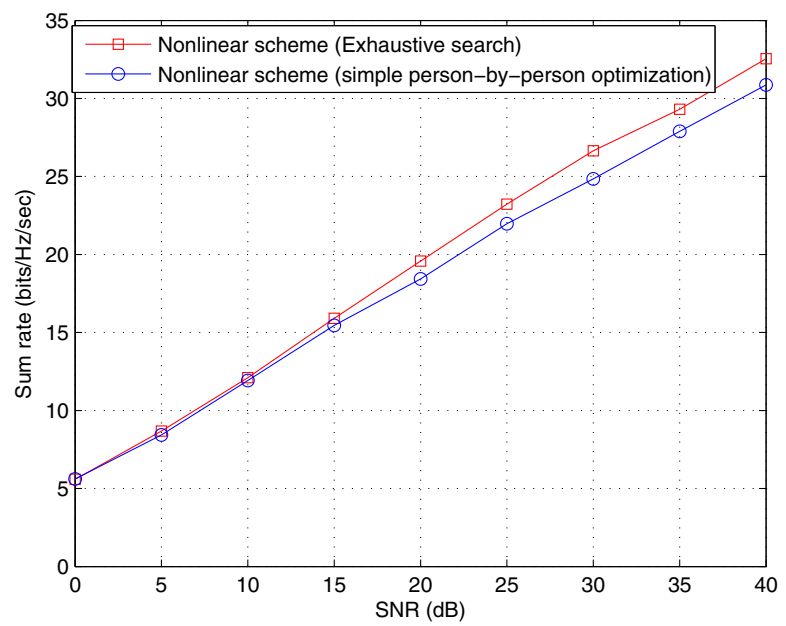

Fig. 5. Sum rates of the proposed scheme with MUD: exhaustive search versus person-by-person optimization $((K, M)=(4,2))$.

can be greatly reduced by simplifying this step. We consider the following person-by-person optimization approach. First, we initialize $\mathcal{S}_{k}$ for receiver $k, k \in \mathcal{K}$, with the indices of randomly chosen $N_{M U D}$ interfering transmitters. We calculate the sum rate for this selection. Then, we pick one receiver and obtain the best set of interfering transmitters for this receiver yielding the highest sum rate while fixing the MUD index sets of the remaining receivers. We move to the next receiver and repeat the same procedure and stop the iteration after some cycles. It will be shown in the next section that this simple person-by-person optimization approach with polynomial complexity performs well compared with the exhaustive search. Thus, the proposed mixed scheme can be designed efficiently.

\section{NUMERICAL RESUlTS}

In this section, we provide some numerical results for the proposed scheme. We considered two overloaded cases $K=4, M=2$ and $K=8, M=4$ satisfying the overloaded 
condition $K \geq 2 M[6]$. For the results in this section, the MIMO channel coefficients of $\mathbf{H}_{k j}$ were generated i.i.d. from complex Gaussian distribution with zero mean and unit variance, and the rate results were obtained by averaging over 500 independent channel realizations. Fig. 3 shows the sum rates of the proposed nonlinear scheme with SIC or MUD, and the linear beamforming scheme. Here, the MaxSINR beamformer design algorithm was used for both cases, and the nonlinear scheme was designed using the person-byperson optimization approach with one cycle. Also, we used $N_{M U D}=1$ in both cases of $K=4$ and $K=8$, based on Proposition 2. As expected, the proposed scheme shows performance with nonzero DoF while the linear scheme shows sum-rate saturation yielding zero DoF. Thus, the performance gain by the proposed mixed scheme over the linear scheme is significant at high SNR in the overloaded case. It is seen that the scheme with MUD yields a higher sum rate than the scheme with SIC, as expected. However, the performance gap is not significant and the DoF is the same for both cases.

Fig. 4 shows the sum rate of the proposed mixed scheme with MUD for several different values of $N_{M U D}$ when $K=4$. As expected by our analysis, the case of $N_{M U D}=1$ yields the highest sum rate. Finally, Fig. 5 shows the performance loss by the simple person-by-person optimization approach. It is seen that the suboptimal person-by-person approach performs almost as well as the exhaustive search.

\section{CONCLUSION}

We have proposed a mixed transceiver architecture based on linear beamforming and MUD to solve the rate saturation problem of purely linear beamforming occurring in overloaded
MIMO interference channels. We have shown that a nontrivial DoF can be achieved by the proposed mixed scheme by properly dividing the interference signals for linear processing and MUD. We have obtained the best design parameters for the proposed mixed scheme and the corresponding DoF. We have provided numerical results to validate the proposed scheme, and the numerical results show that the proposed scheme outperforms linear beamforming in overloaded MIMO interference channels. The proposed mixed scheme provides an alternative to handle overloaded MIMO interference systems when scheduling among the transmitters is not available.

\section{REFERENCES}

[1] V. R. Cadambe and S. A. Jafar, "Interference alignment and degrees of freedom of the $K$-user interference channel," IEEE Trans. Inf. Theory, vol. 54, no. 8, pp. 3425-3441, Aug. 2008.

[2] K. Gomadam, V. R. Cadambe, and S. A. Jafar, "A distributed numerical approach to interference alignment and application to wireless interference networks," IEEE Trans. Inf. Theory, vol. 57, no. 6, pp. 3309-3322, June 2011.

[3] S. W. Peters and R. W. Heath, Jr., "Cooperative algorithms for MIMO interference channels," IEEE Trans. Veh. Technol., vol. 60, no. 1, pp. 206-218, Jan. 2011.

[4] H. Yu and Y. Sung, "Least squares approach to joint beam design for interference alignment in multiuser multi-input multi-ouput interference channels," IEEE Trans. Signal Process., vol. 58, no. 9, pp. 4960-4966, Sep. 2010.

[5] J. Park, Y. Sung, and H. V. Poor, "On beamformer design for multiuser MIMO interference channels," ArXiv pre-print cs.IT/0153879, Nov. 2010.

[6] C. M. Yetis, T. Gou, S. A. Jafar, and A. H. Kayran, "On feasibility of interference alignment in MIMO interference networks," IEEE Trans. Signal Process., vol. 58, no. 9, pp. 4771-4782, Sep. 2010.

[7] T. S. Han and K. Kobayashi, "A new achievable rate region for the interference channel," IEEE Trans. Inf. Theory, vol. IT-27, pp. 49-60, Jan. 1981.

[8] T. M. Cover and J. A. Thomas, Elements of Information Theory. John Wiley \& Sons, Inc., 2006. 